\title{
The effectiveness of $\mathrm{MgCeAl}_{11} \mathrm{O}_{19}: \mathrm{Tb}^{3+}$ phosphor in enhancing the luminous efficacy and color quality of multi-chip white LEDs
}

\author{
Nguyen Thi Phuong Loan', Nguyen Doan Quoc Anh' \\ ${ }^{1}$ Faculty of Fundamental 2, Posts and Telecommunications Institute of Technology, Vietnam \\ ${ }^{2}$ Power System Optimization Research Group, Faculty of Electrical and Electronics Engineering, \\ Ton DucThang University, Vietnam
}

\begin{tabular}{l} 
Article Info \\
\hline Article history: \\
Received Nov 13, 2019 \\
Revised Mar 13, 2020 \\
Accepted Mar 27, 2020 \\
\hline
\end{tabular}

\section{Keywords:}

Color quality scale Luminous flux MgCeAl11019:Tb3+ Mie-scattering theory WLEDs

\begin{abstract}
In this research paper, we introduced yellow-green $\mathrm{MgCeAl}_{11} \mathrm{O}_{19}: \mathrm{Tb}^{3+}$ as a new phosphor ingredient to adapt to the quality requirements on the chromatic homogeneity and emitted luminous flux of modern multi-chip white LED lights (MCW-LEDs). The results from experiments and simulation show that employing $\mathrm{MgCeAl}_{11} \mathrm{O}_{19}: \mathrm{Tb}^{3+}$ phosphor can lead to much better optical properties and therefore is a perfect supporting material to achieve the goals of the research. When the $\mathrm{MgCeAl}_{11} \mathrm{O}_{19}: \mathrm{Tb}^{3+}$ phosphor is added into the phosphorus composite which already contains YAG: $\mathrm{Ce}^{3+}$ particles, and the silicone glue, it affects the optical properties significantly. In other words, the concentration of this phosphor can determine the efficiency of lumen output and chromatic homogeneity of WLEDs. In specific, as the concentration of $\mathrm{MgCeAl}_{11} \mathrm{O}_{19}: \mathrm{Tb}^{3+}$ go up, the luminous yield will increase accordingly, though there is an insignificant decrease in CQS. Moreover, if the $\mathrm{MgCeAl}_{11} \mathrm{O}_{19}: \mathrm{Tb}^{3+}$ concentration reduce a little bit, it is possible to better the correlated color temperature uniformity and lumen efficacy of LED packages. In addition, the Mie scattering theory, Monte Carlo simulation and LightTools 8.3.2 software are employed to analyze and simulate the LED packages' structure as well as the phosphor compound.
\end{abstract}

Copyright (C) 2020Institute of Advanced Engineering and Science. All rights reserved.

\section{Corresponding Author:}

Nguyen Doan Quoc Anh,

Power System Optimization Research Group,

Faculty of Electrical and Electronics Engineering,

Ton Duc Thang University,

No. 19 Nguyen HuuTho Street, Tan Phong Ward, District 7, Ho Chi Minh City, Vietnam.

Email: nguyendoanquocanh@tdtu.edu.vn

\section{INTRODUCTION}

White light-emitting diodes (WLEDs) are considered as a potential light source that can fulfill the fastidious illumination market just because they are advantageous in compact size, energy saving, eco-friendly, and long lifespan. This also the reason why WLEDs havea widespread application over the human life thesedays, from backlighting to street and indoor lightings [1-3]. Up to now, the method used to producecommercial phosphor-converted WLEDs is combining the blue LED chips and yellow $\mathrm{Y}_{3} \mathrm{Al}_{5} \mathrm{O}_{12}: \mathrm{Ce}^{3+}\left(\mathrm{YAG}: \mathrm{Ce}^{3+}\right.$ ) phosphor embedded with organic resins [4, 5]. Nevertheless, when the organic encapsulants is applied to high-power WLEDs, it shows poor thermal and photonic stability, may wane easily and turns yellow after using for a long time. As a result, this degradation probably reduces the luminous efficacy (LE), the chromatic quality, and the long-term reliability [6, 7]. Moreover, it is showed that compared to the YAG:Ce ${ }^{3+}$ phosphor, which has refractive index at about 1.83 , that of the organic encapsulants is lower as its value is approximately 1.5. This difference causes the reflection loss to happen and as a result, the emitted lights from phosphor particles are downgraded $[8,9]$. 
Owing to that disadvantage of organic encapsulants, an alternative approach called phosphor-in-glass (PiG) was proposed. To prepare PiG, glass powders and phosphor particles are sintered at a low-temperature $\left(<800^{\circ} \mathrm{C}\right)$. Researchers considered $\mathrm{PiG}$ as a potential luminescent converter for high-power WLEDs as it has the robustness, high thermal stability, and low thermal expansion coefficient [10-15]. Moreover, it is possible for the precursor glass matrix to accomplish a high refractive index for the PiG converter by addinghigh polarizable ions. Last year, we succeeded in developing YAG:Ce ${ }^{3+}$ based $\mathrm{PiG}$ converter by embedding the yellow phosphor YAG:Ce ${ }^{3+}$ with borosilicate glassusing screen-printing and low-temperature sintering methods. The product can yield cool white light with the values of lumen efficacy,correlated color temperature (CCT), and color rendering index (CRI) of $114 \mathrm{~lm} / \mathrm{W}, 5524 \mathrm{~K}$ and 69, respectively [16, 17]. However, this YAG: $\mathrm{Ce}^{3+} \mathrm{PiG}$ does not generate true white light having high CRI because the emission light is short of red light spectrum. To fulfill the goal of enhancing the CRI, researchers proposed multi-components $\mathrm{PiG}$, which is invented by having red $\mathrm{CaAlSiN}_{3}: \mathrm{Eu}^{2+}\left(\mathrm{CASN}: \mathrm{Eu}^{2+}\right)$ phosphor added into the glass matrix [18-20]. Yet, there are noticeable drawbacks of this PiG: the effect of thermal degradation during co-sintering on it, and the interfacial reaction it caused to the glass matrix. These influences probably decrease the quantum efficiency of red phosphor [21,22]. Additionally, when using the PiG converter with flat surface, the total internal reflection (TIR) at the glass-air interface also exits as the refractive indexes of glass and air are different; and as a result, the loss of light trapped in the remote-type LED package occurs.

For the aim of TIR loss reduction and light extraction enhancement, the micro/nano patterned structures were widely applied. Currently, there have been many strategies suggested to produce the patterned structures consisting lithography [23], photoresist reflow, direct laser writing, and inkjet imprinting [24]. But in fact, most of the processes in those strategies consist of at least one photolithographic step which requires much money and time to do as well as leads to heavy pollution. It is possible to produce high precise patterned structure by direct laser writing, but it is high-cost and impracticalfor mass production. Another method proposed to cover the disadvantages is inkjet printingowing to its advantages in production which are mask-less, large-scale, and low-cost. However, the drawback of this method is the limitation caused by inhomogeneous morphology and large feature size. Hence, to successfully produce the YAG:Ce ${ }^{3+}$ $\mathrm{PiG}$ with high light extraction and color quality, there is a big challenge in choosing the most flexible and effective approach to apply. On the hand, choosing the suitable WLED configuration is also an important decision as it affect the quality evaluation of WLED. Regarding this subject, there are many research have proposed different alterations that achieved certain improvements. For example, the remote phosphor structure is proposed for utilization in many phosphor structures to increase the color quality of White LEDs. There are reports that claimed the two essential quality indicators of WLED, CRI and lumen output, can be enhanced with the application convex-dual-layer remote phosphor geometry containing the red-emitting phosphor $\mathrm{Mg}_{8} \mathrm{Ge}_{2} \mathrm{O}_{11} \mathrm{~F}_{2}: \mathrm{Mn}^{4+}$. The remote phosphor structure with flat dual phosphor layers are also reported to be efficient in enhancing WLED, especially the luminous flux. However, these research concentrate on the light output or CRI while there are other more important optical properties. Moreover, the in-cup phosphor geometry (IGP) and conformal phosphor geometry (CPG) were not mentioned and the phosphor utilized in the experiments is the red-emitting phosphor. Thus, this research come up with an approach which utilize the green phosphor as a new phosphor material and including other aspects of WLED such as scattering coefficient and CQS. The influences of the green phosphor on the optical properties of WLED will be estimated by changing the phosphor concentration.

In this article, the yellow-green $\mathrm{MgCeAl}_{11} \mathrm{O}_{19}: \mathrm{Tb}^{3+}$ phosphor is used as a support of increasing chromatic homogeneity and emitted luminous flux to achieve the greatest efficiency in the phosphor compounding of MCW-LEDs during the scattering process. Specifically, the presence of $\mathrm{MgCeAl}_{11} \mathrm{O}_{19}$ : $\mathrm{Tb}^{3+}$ particles is capable of determining the illuminating quality and chromatic homogeneity. The research is comprised of 3 stages: (1) Building the MCW-LED model; (2) Adding $\mathrm{MgCeAl}_{11} \mathrm{O}_{19}$ : $\mathrm{Tb}^{3+}$ to the phosphor compounding used for the simulated MCW-LEDs; (3) Investigating how the concentration of $\mathrm{MgCeAl}_{11} \mathrm{O}_{19}: \mathrm{Tb}^{3+}$ impactthe emission spectra and the scattering of the structure. The research will calculated the emission spectra, scattering coefficient, lumen output, and CQS of WLED with $\operatorname{MgCeAl}_{11} \mathrm{O}_{19}: \mathrm{Tb}^{3+}$ in conformal phosphor and in-cup phosphor configuration. The mathematical equations for the optical properties calculation are also presented in this paper. Briefly, combining $\mathrm{MgCeAl}_{11} \mathrm{O}_{19}: \mathrm{Tb}^{3+}$ together with YAG: $\mathrm{Ce}^{3+}$ phosphors is considered as an optimal approach to push the color uniformity and lumen output to the highest value. This research can provide information and support the manufacturers in identify the suitable configuration to fabricate high quality WLED.

\section{PREPARATION AND SIMULATION}

\subsection{Preparation of yellow-green $\mathrm{MgCeAl}_{11} \mathrm{O}_{19}: \mathrm{Tb}^{3+}$ phosphor}

To achieve the optimal $\mathrm{MgCeAl}_{11} \mathrm{O}_{19}: \mathrm{Tb}^{3+}$ phosphor, it is important to get the preparation carried out in a strict order. The preparation process will go through six steps described as follows. Initially, the materials are 
mixed by using an automatic mill. Next, the mixed product is taken into the alumina crucibles and fired with stagnant ait at the temperature of $1650^{\circ} \mathrm{C}$ in 2 hours. After that, powderizing the material by automatic mill and re-firing it in alumina boats at $1600^{\circ} \mathrm{C}$ in the same time as the first firing. However, for the re-firing stage, it is vital to reduce the atmosphere $\left(95 \% \mathrm{~N}_{2} / 5 \% \mathrm{H}_{2}\right)$. Then, the fired mixture is powderized again. Finally, the obtained product will be stored in a well-sealed container.

\subsection{Construction of MC-WLEDs}

To construct a conventional phosphor-conversion white LED, it is essential to dispense the compounding made of transparent silicone binder and phosphor grains into a die-bonded lead frame. However, inside this LED model, the opportunity that the blue lights reach the phosphor particles is diverse considerably depending on different light angle, which leads to the low angular color uniformityof the package [7-9]. Thus, the purpose here is to enhance the chromatic homogeneity of the LED packages. Then, researchers came up with the remote phosphor LED package including a phosphor layer or plate to attain this goal [10-12]. Hence, this study is carried out with the application of the remote phosphor structure so that we can achievea emitting surface that is uniform with a rectangular shape on an LED package and fulfill the requirement of the ISO 9680 regulation.

According to Figure 1, the remote phosphor LED package was simulated by applying the LightTools 8.3.2 software with the support of Monte Carlo ray tracing. The two LED chips are placed horizontally on the bottom of the reflector cup. Theirwidth, length, and height are $1.33 \mathrm{~mm}, 1.33 \mathrm{~mm}$, and $0.17 \mathrm{~mm}$, respectively.For the cavity of the LED (width/length/height $=6 / 5 / 2.8 \mathrm{~mm}$ ), its dimensions are width $=44 \mathrm{~mm}$, length $=3.06 \mathrm{~mm}$, and depth $=1.7 \mathrm{~mm}$. In addition, the bottom of the reflector cup was seen as a mirror with the reflectivity of $90 \%$. Meanwhile, the side surfaces get their reflective properties followed Gaussian scattering with $90 \%$ reflectivity and $10^{\circ}$ Gaussian spread. Besides, the transparent glass substrate covered by a phosphor layeris located at the top surface of the reflector cup to protect the LED chips. Besides, the glass substrate and phosphor film whose width and length are the same $(5 \mathrm{~mm} \times 3.5 \mathrm{~mm})$ have the thickness of $0.2 \mathrm{~mm}$ and $0.15 \mathrm{~mm}$, in turn.

Inside the LED lamp, just a proportion of blue lights from LED chips can directly get to the interface between the glass substrate and the phosphor layer, while the others have to going through a process of reflection caused by the reflector cup before arriving at the destination. Besides, the structure of the reflector cup and the gap between two LED chips were amended so as to obtain the homogeneous illuminance at the interface. After passing the glass substrate, the blue light will be absorbed by phosphor grains or scattered without any absorption, when they reach the phosphor film. Additionally, the Stokes shift can have some absorbed blue lights down-converted to green-red light. Meanwhile, the unconverted light is released as a non-radiative loss. Finally, the white light is generated at the top emitting surface by the combination of green-red light and blue light which are converted and unabsorbed, respectively. For the phosphor film, it consists of transparent silicone binder, green (LuAG:Ce) and red (CASN:Eu) phosphors to reach the CRI over 90, in which the LuAG:Ce phosphor and CASN:Eu phosphor have the phosphor density of $6 \mathrm{~g} / \mathrm{cm}^{3}$ and $3.1 \mathrm{~g} / \mathrm{cm}^{3}$, the average particle size of approximate $13 \mu \mathrm{m}$ and $12 \mu \mathrm{m}$, respectively. The excitation, quantum yield, and emission spectra of each phosphor are verified by employing a quantum yield measurement framework. The parameters of phosphors were updated to the optical configuration which then simulated with the Monte Carlo ray tracing and Mie scattering.

Figure 1(c) illustrated the conformal phosphor structure (CPG), and Figure 1(d) presented the in-cup phosphor structure (IPG). From Figure 1(c), we can observe that the proposed phosphor compounding covers the chips conformally. In contrast, for the in-cup phosphor structure and for mixing with the silicone lens, the methods of separation are applied. Then, the Mie-theory is used to analyse the scattering processes of these two structures. In addition, the mean phosphor particles diameter adopted in this research is $14.5 \mu \mathrm{m}$ which is equal to the actual parameter.

The phosphor compounding used in the experiments is comprised of $\mathrm{MgCeAl}_{11} \mathrm{O}_{19}: \mathrm{Tb}^{3+}$ particles, YAG: $\mathrm{Ce}^{3+}$ particles, and the silicone glue that are completely mixed together. Moreover, the sizes of these elements are the similar to the actual ones whose refractive indexes are respectively of 1.85, 1.83, and 1.52. After verifying the refractive index and the size of the phosphor grains, it is possible to determine the emission spectra of phosphor mixture. Figure 2 demonstrates the emission spectra of two phosphor structures along with different concentration values of $\mathrm{MgCeAl}_{11} \mathrm{O}_{19}: \mathrm{Tb}^{3+}$ : (a) the conformal with $\mathrm{MgCeAl}_{11} \mathrm{O}_{19}: \mathrm{Tb}^{3+}$ concentration from $0 \%$ to $4 \%$, and (b) the in-cup with $\mathrm{MgCeAl}_{11} \mathrm{O}_{19}: \mathrm{Tb}^{3+}$ varying from $0 \%$ to $1.4 \%$ sometimes. Based on this chart, the luminous flux of MCW-LEDs can be promoted as soon as the $\mathrm{MgCe}_{11} \mathrm{O}_{19}: \mathrm{Tb}^{3+}$ phosphor is added into the phosphor compound. 


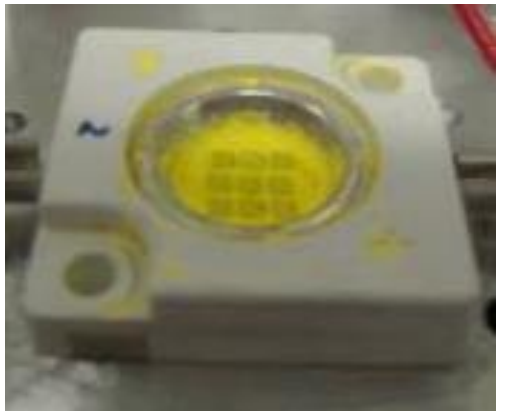

(a)

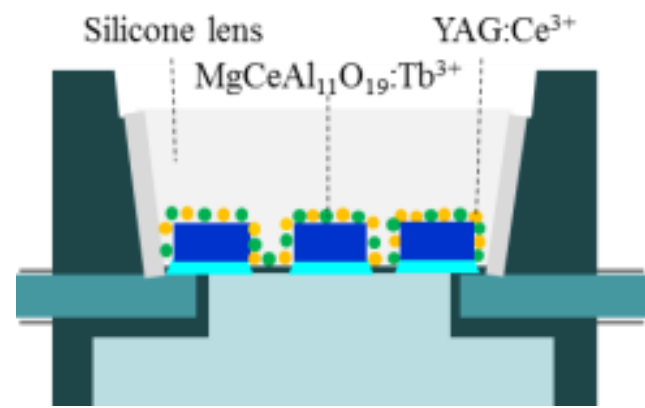

(c)
Lead frame: $4.7 \mathrm{~mm}$ Jentech Size-S

LED chip: V45H

Die attach: Sumitomo 1295SA

Gold Wire: 1.0 mil

Phosphor: ITC NYAG4_EL

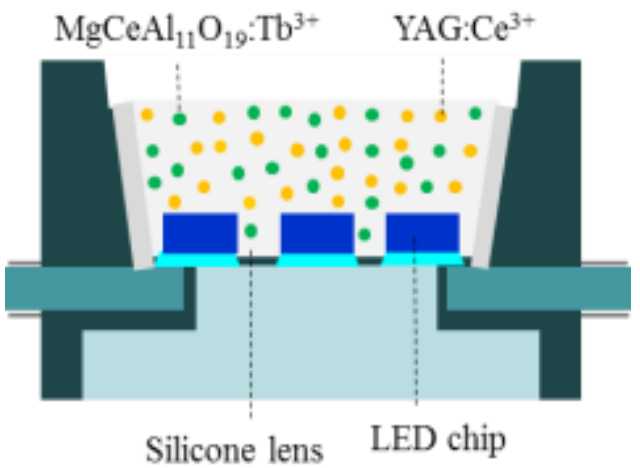

(d)

Figure 1. Illustration of phosphor-converted MCW-LEDs as doping $\mathrm{MgCeAl}_{11} \mathrm{O}_{19}: \mathrm{Tb}^{3+}$ : (a) The actual MCWLEDs and (b) its parameters; (c) Conformal phosphor geometry (CPG); (d) In-cup phosphor geometry (IPG)

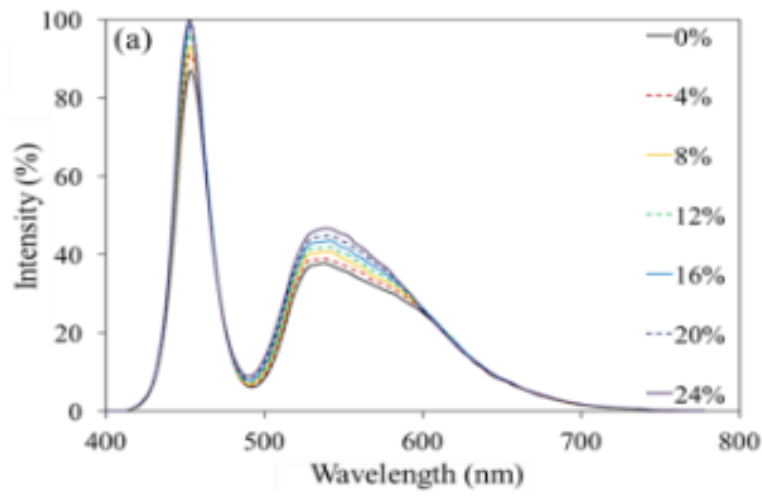

(a)

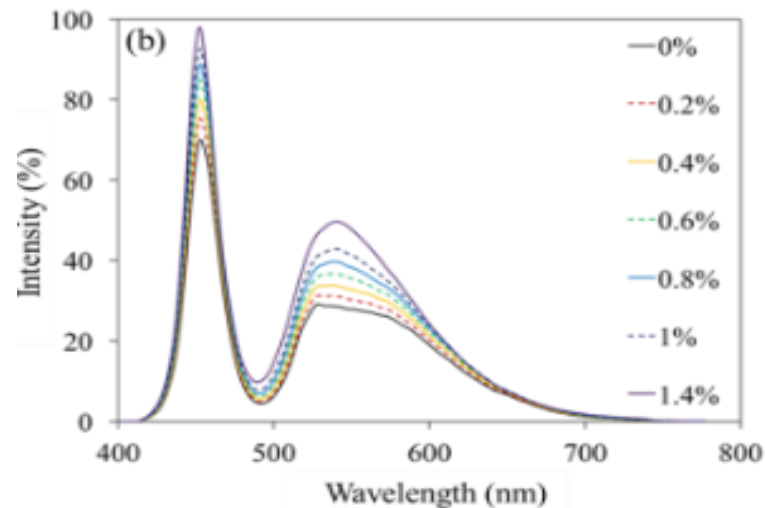

(b)

Figure 2. Emission spectra of multiple phosphors: (a) CPG and (b) IPG

\section{COMPUTATION AND DISCUSSION}

In order to confirm the optical properties of phosphor compounding, the scattering coefficient $\mu_{s c a}$ are calculated based on the Mie theory [25]. The following expressions demonstrate the connection among the three elements: the scattering coefficient (SC), the wavelength, and the size of $\mathrm{MgCeAl}_{11} \mathrm{O}_{19}$ : $\mathrm{Tb}^{3+}$ particles.

$$
\begin{aligned}
& \mu_{s c a}(\lambda)=\frac{c}{\bar{m}} \bar{C}_{s c a}(\lambda) \\
& \bar{C}_{s c a}(\lambda)=\frac{\int C_{s c a, D}(\lambda) f(\mathrm{D}) \mathrm{dD}}{\int f(\mathrm{D}) \mathrm{dD}}
\end{aligned}
$$




$$
\begin{gathered}
\bar{m}=\frac{\int m_{i}(\mathrm{D}) f(\mathrm{D}) \mathrm{dD}}{\int f(\mathrm{D}) \mathrm{dD}} \\
C_{s c a}(\lambda)=\frac{P_{s c a}(\lambda)}{I_{i n c}(\lambda)}
\end{gathered}
$$

In these expressions, $f(D)$ indicates the size distribution function, $c$ is the phosphor concentration $\left(\mathrm{g} / \mathrm{cm}^{3}\right)$, and $C_{s c a, D}$ is the scattering cross-section of the phosphor with the particle diameter $D$. Besides, $\bar{C}_{s c a}(\lambda)$ and $\bar{m}$ present the scattering cross-section and the particle mass of the phosphor integrated over $f(D)$, respectively. Additionally, $P_{\text {sca }}(\lambda)$ is known as the scattered power by phosphor particles, while $I_{\text {inc }}(\lambda)$ are the irradiance intensity. Figure 3 shows the scattering coefficient (SC) of the phosphor layer as the $\mathrm{MgCeAl}_{11} \mathrm{O}_{19}: \mathrm{Tb}^{3+}$ phosphor participates in the scattering process.In fact, the concentrations and other factors of $\mathrm{MgCeAl}_{11} \mathrm{O}_{19}$ : $\mathrm{Tb}^{3+}$ can cause the $\mathrm{SC}$ of phosphorus mixture to change greatly. Additionally, this conclusion also assures the impacts of previous concentrations and stimulation of $\mathrm{MgCeAl}_{11} \mathrm{O}_{19}: \mathrm{Tb}^{3+}$ on the color quality of both CPG and IPG structures. As can be seen, the increase in $\mathrm{MgCeAl}_{11} \mathrm{O}_{19}: \mathrm{Tb}^{3+}$ concentration leads to the upward tendency of SC no matter what particle size of $\mathrm{MgCeAl}_{11} \mathrm{O}_{19}: \mathrm{Tb}^{3+}$. Moreover, when the $\mathrm{MgCeAl}_{11} \mathrm{O}_{19}: \mathrm{Tb}^{3+}$ phosphor particle is set at about $1 \mu \mathrm{m}, \mathrm{SC}$ can reach the highest value and better the chromatic uniformity, without consideration for the larger sizes. Meanwhile, with the size of approximately $7 \mu \mathrm{m}$, the SC value is more stable, which is independent of the rise in $\mathrm{MgCeAl}_{11} \mathrm{O}_{19}: \mathrm{Tb}^{3+}$ concentration. As a result, with this size, the color quality (CQS) of LED lights is advanced. Thus, if the goal is to improve the CQS, the size of $\mathrm{MgCeAl}_{11} \mathrm{O}_{19}$ : $\mathrm{Tb}^{3+}$ should be at $1 \mu \mathrm{m}$ and less than $7 \mu \mathrm{m}$. Obviously, the concentration and the size of $\mathrm{MgCeAl}_{11} \mathrm{O}_{19}: \mathrm{Tb}^{3+}$ can determine the SC value. That's why $\mathrm{MgCeAl}_{11} \mathrm{O}_{19}: \mathrm{Tb}^{3+}$ can be employed to lift up the luminous efficiency and color uniformity of the LEDs.

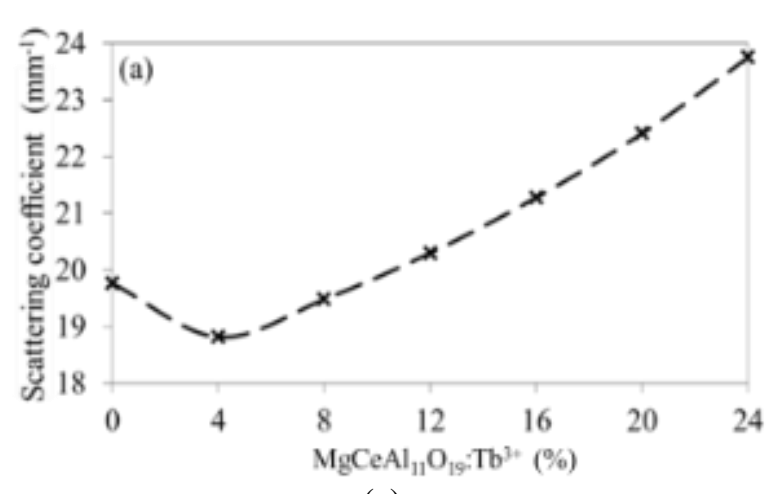

(a)

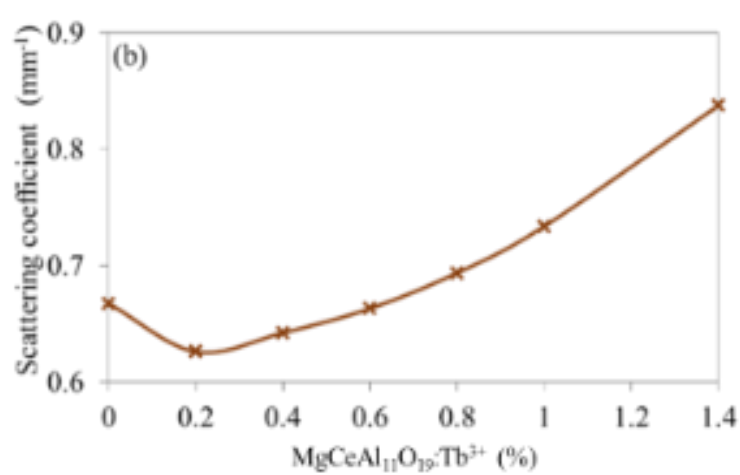

(b)

Figure 3. Scattering coefficients of phosphor compounding at $453 \mathrm{~nm}$ as a function of the concentration and size of $\mathrm{MgCeAl}_{11} \mathrm{O}_{19}: \mathrm{Tb}^{3+}$ : (a) CPG and (b) IPG

The essential purpose that needs to be accomplished in this article is fulfilling the requirements of the LED product specification. Thus, the ACCT that needs for the effective operation of MCW-LED is in the range of $8500 \mathrm{~K}$. In addition, as the $\mathrm{MgCeAl}_{11} \mathrm{O}_{19}: \mathrm{Tb}^{3+}$ phosphor concentration increases, the yellow YAG: $\mathrm{Ce}^{3+}$ phosphor concentration simultaneously decreases to keep the ACCT at $8500 \mathrm{~K}$.The weight percentage of the LED phosphor layer is presented in the following formula.

$$
\sum W_{p l}=W_{\text {yellow phosphor }}+W_{\text {silicone }}+W_{\text {yellow-green phosphor }}=100 \%
$$

where $W_{\text {silicone }}, W_{\text {yellow phosphor }}$ and $W_{\text {yellow-green phosphor }}$ representthe weight percentage of the silicone glue, the yellow YAG:Ce ${ }^{3+}$ phosphor, and the yellow-green $\mathrm{MgCeAl}_{11} \mathrm{O}_{19}$ : $\mathrm{Tb}^{3+}$ phosphor, respectively.

When the $\mathrm{MgCeAl}_{11} \mathrm{O}_{19}: \mathrm{Tb}^{3+}$ is added to the phosphor layer, the color deviation of MCW-LED is affected, as demonstrated in Figure 4. Obviously, the presence of $\mathrm{MgCeAl}_{11} \mathrm{O}_{19}$ : $\mathrm{Tb}^{3+}$ will probably cause the CCT peak deviation todecline noticeably. In other words, the spatial distribution of MCW-LED with $\mathrm{MgCeAl}_{11} \mathrm{O}_{19}: \mathrm{Tb}^{3+}$ is much flatter than the absence of $\mathrm{MgCeAl}_{11} \mathrm{O}_{19}: \mathrm{Tb}^{3+}$. 

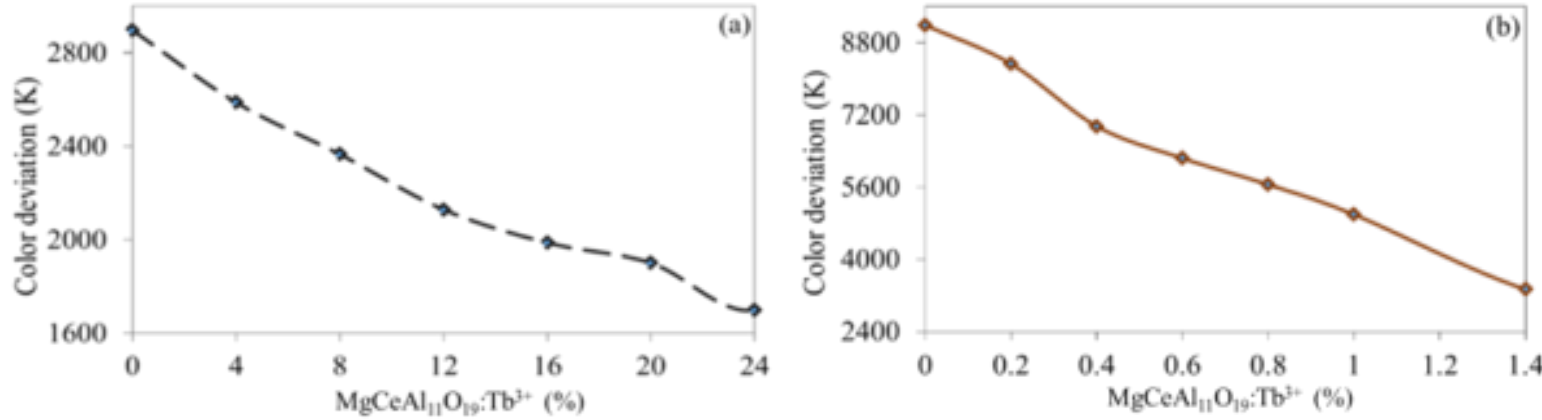

Figure 4. The CCT peak-valley deviation as a function of the concentration and size of $\mathrm{MgCeAl}_{11} \mathrm{O}_{19}: \mathrm{Tb}^{3+}$ : (a) $\mathrm{CPG}$; (b) IPG

To acquire the high-quality LED products, it is crucial to focus on both performance factors and optimization issues since the optical systems will not be fully optimized if we just concentrate on promoting a single element. In other words, the WLEDs cannot accomplish the highest CQS and efficiency at the same time. Thus, to be able to solve this problem, the spectrum and wide source efficiency must be maximized in monochromatic radiation of $555 \mathrm{~nm}$ wavelength to enhance CRI. In this paper, CQS, luminous flux, and CCT $\mathrm{P}-\mathrm{V}$ deviation values are put into comparison.

From the attained results illustrated in Figures 5 and 6 , it is possible to say that the growth of luminous yield is compatible with the increase of $\mathrm{MgCeAl}_{11} \mathrm{O}_{19}$ : $\mathrm{Tb}^{3+}$ concentration. Though the higher concentration of $\mathrm{MgCeAl}_{11} \mathrm{O}_{19}: \mathrm{Tb}^{3+}$ will better the luminous flux, the CQS will decline. Besides, when the $\mathrm{MgCeAl}_{11} \mathrm{O}_{19}: \mathrm{Tb}^{3+}$ concentration decrease but insignificantly, the uniformity of correlated color temperature and lumen efficiency can be enhanced significantly.
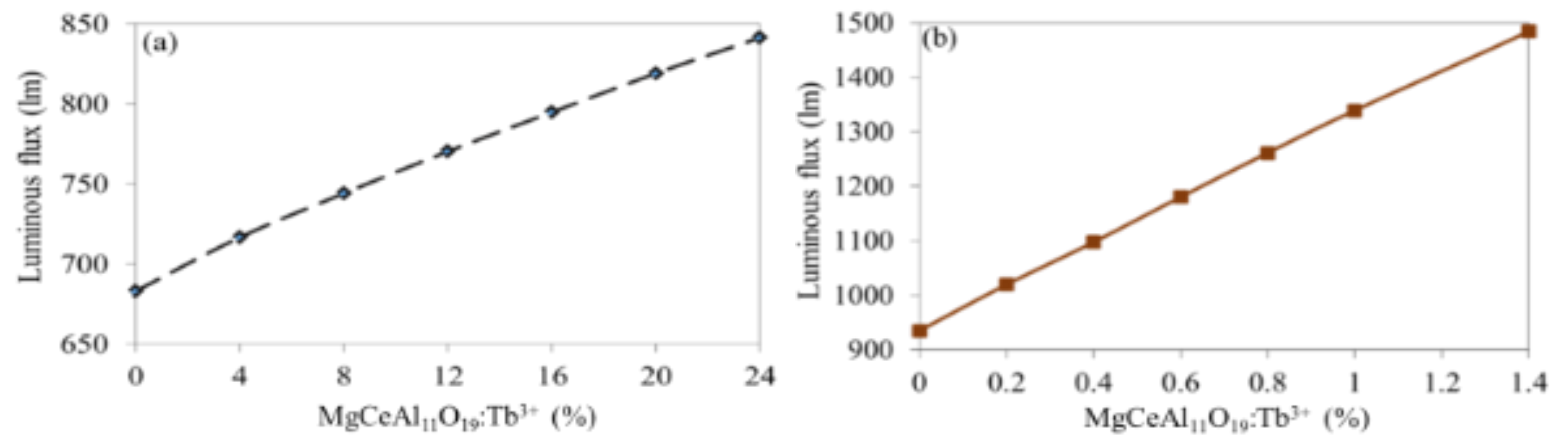

Figure 5. Luminous efficacy as a function of the concentration and size of $\mathrm{MgCeAl}_{11} \mathrm{O}_{19}: \mathrm{Tb}^{3+}$ :

(a) CPG and (b) IPG
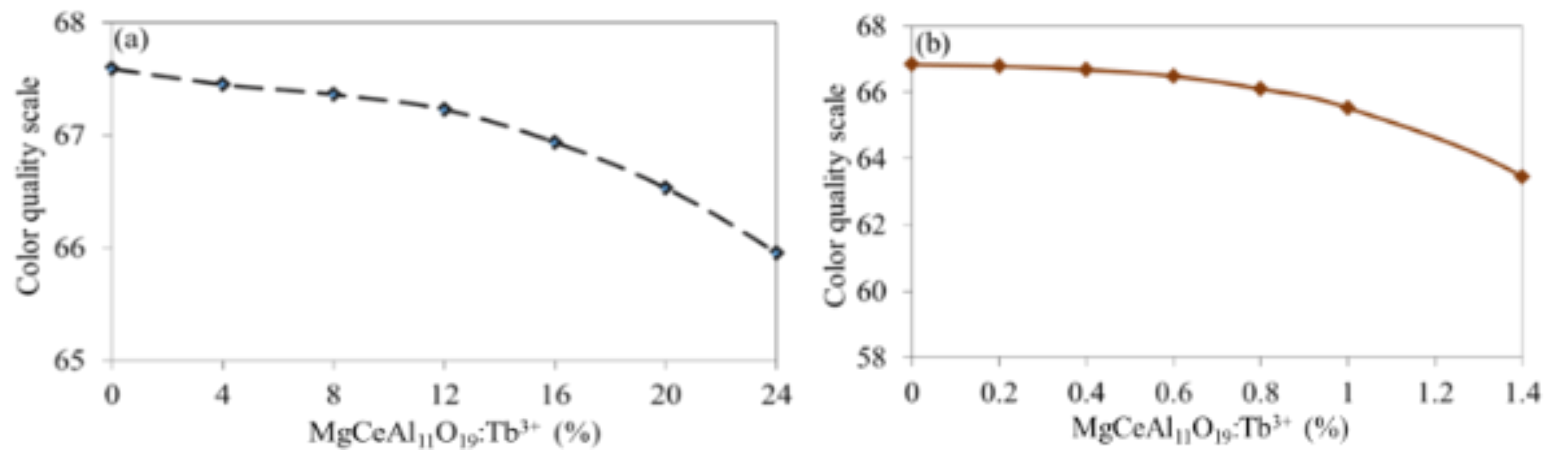

Figure 6. Color quality scale as a function of the concentration and size of $\mathrm{MgCeAl}_{11} \mathrm{O}_{19}: \mathrm{Tb}^{3+}$ :

(a) CPG; (b) IPG 


\section{CONCLUSION}

To further enhance the optical characteristics of modern multi-chip white LED lamps (MCW-LEDs) for application inmore advanced and demanding utilization. Inshort, through results of experiments and simulations of this study, the yellow-green $\mathrm{MgCeAl}_{11} \mathrm{O}_{19}: \mathrm{Tb}^{3+}$ has proved its effectiveness in getting the color quality and lumen output of WLEDsimproved. There are two noticeable results that reinforce the efficiency of employing the yellow-green $\mathrm{MgCeAl}_{11} \mathrm{O}_{19}: \mathrm{Tb}^{3+}$ phosphor. First, with the application of Mie scattering theory, it demonstrates that the compensation of light scattering for WLEDs leads to the diversity in color uniformity, regardless of ACCT values. Second, the Monte Carlo simulation was applied to compute the lumen output which result showed significant improvement when the amount of $\mathrm{MgCeAl}_{11} \mathrm{O}_{19}$ : $\mathrm{Tb}^{3+}$ concentration increased. One small notice is that the high concentration in $\mathrm{MgCeAl}_{11} \mathrm{O}_{19}: \mathrm{Tb}^{3+}$ can negatively affect the value of CQS, an important quality indicator for WLED, therefore, keeping $\mathrm{MgCe} \mathrm{Al}_{11} \mathrm{O}_{19}$ : $\mathrm{Tb}^{3+}$ concentration under a certain percent that does not damage the CQS is most advisable. However, with the superior values achieved in different optical qualities when this type of phosphor is applied compared to previous phosphor configurations, $\mathrm{MgCeAl}_{11} \mathrm{O}_{19}: \mathrm{Tb}^{3+}$ phosphor is the still the more advanced choicefor developing phosphorus materials and manufacturing white LED packages. The manufacturers can refer to the results of this research as guideline for the production of high-quality LED or references for further quality development study.

\section{REFERENCES}

[1] S. K. Abeysekera, et al., "Impact of circadian tuning on the illuminance and color uniformity of a multichannel luminaire with spatially optimized LED placement," Optics Express, vol. 28, pp. 130-145, 2020.

[2] N. D. Q. Anh, et al., "Selection of a remote phosphor configuration to enhance the color quality of white LEDs," Current Optics and Photonics, vol. 3, pp. 78-85, 2019.

[3] Y. Peng, et al., "Flexible fabrication of a patterned red phosphor layer on a YAG:Ce3+ phosphor-in-glass for high-power WLEDs," Optical Materials Express, vol. 8, pp. 605-614, 2018.

[4] H. Lee, et al., "Phosphor-in-glass with Nd-doped glass for a white LED with a wide color gamut," Optics Letters, vol. 43, pp. 627-630, 2018.

[5] A. K. Dubey, et al., "Laser-line-driven phosphor-converted extended white light source with uniform illumination," Applied Optics, vol. 58, pp. 2402-2407, 2019.

[6] G. Zhang, et al., "Spectral optimization of color temperature tunable white LEDs with red LEDs instead of phosphor for an excellent IES color fidelity index," OSA Continuum, vol. 2, pp. 1056-1064, 2019.

[7] J. Hao, et al., "Prediction of lifetime by lumen degradation and color shift for LED lamps, in a non-accelerated reliability test over 20,000 h," Applied Optics, vol. 58, pp. 1855-1861, 2019.

[8] C. W. Zhang, et al., "Photometric optimization and comparison of hybrid white LEDs for mesopic road lighting," Applied Optics, vol. 57, pp. 4665-4671, 2018.

[9] A. A. Alatawi, et al., "High-power blue superluminescent diode for high CRI lighting and high-speed visible light communication," Optics Express, vol. 26, pp. 26355-26364, 2018.

[10] J. F. Ruan, et al., "Phase transformation induced reversible modulation of upconversion luminescence of WO3:Yb3+,Er3+ phosphor for switching devices," Optics Letters, vol. 43, pp. 3885-3888, 2018.

[11] B. Li, et al., "High-efficiency cubic-phased blue-emitting Ba3Lu2B6O15:Ce3+ phosphors for ultraviolet-excited white-light-emitting diodes," Optics Letters, vol. 43, pp. 5138-5141, 2018.

[12] X. Wang, et al., "Broadband multicolor upconversion from Yb3+-Mn2+codoped fluorosilicate glasses and transparent glass ceramics," Optics Letters, vol. 43, pp. 5013-5016, 2018.

[13] Y. Yuan, et al., "High luminous fluorescence generation using Ce:YAG transparent ceramic excited by blue laser diode," Optical Materials Express, vol. 8, pp. 2760-2767, 2018.

[14] S. R. Chung, et al., "Full color display fabricated by CdSe bi-color quantum dots-based white light-emitting diodes," Optical Materials Express, vol. 8, pp. 2677-2686, 2018.

[15] F. Steudel, et al., "Pixelated phosphors for high-resolution and high-contrast white light sources," Optics Express, vol. 26, pp. 26134-26144, 2018.

[16] L. Tang, et al., "Photo-induced luminescence degradation in $\mathrm{Ce}, \mathrm{Yb}$ co-doped yttrium aluminum garnet phosphors," Applied Optics, vol. 57, pp. 7627-7633, 2018.

[17] R. Lei, et al., "Influence of excitation power and doping concentration on the upconversion emission and optical temperature sensing behavior of Er3+:BaGd2(MoO4)4 phosphors," Optical Materials Express, vol. 8, pp. 3023-3035, 2018.

[18] X. Huang, et al., "High-efficiency and thermally stable far-red-emitting NaLaMgWO6:Mn4+phosphorsfor indoor plant growth light-emitting diodes," Optics Letters, vol. 43, pp. 3305-3308, 2018.

[19] V. V. Bakhmet'ev, et al., "Effect of copper introduction and shock-wave processing of zinc sulfide on the spectral characteristics of a manganese-activated phosphor synthesized from it," Journal of Optical Technology, vol. 85, pp. 367-370, 2018.

[20] X. L. Song, et al., "Biaxial hyperbolic metamaterials using anisotropic few-layer black phosphorus," Optics Express, vol. 26, pp. 5469-5477, 2018.

[21] J. Cao, et al., "Upconversion luminescence of $\mathrm{Ba} 3 \mathrm{La}(\mathrm{PO} 4) 3: \mathrm{Yb} 3+-\mathrm{Er} 3+/ \mathrm{Tm} 3+$ phosphors for optimal temperature sensing," Applied Optics, vol. 57, pp. 1345-1350, 2018. 
[22] T. Wei, et al., "Single Pr3+-activated high-color-stability fluoride white-light phosphor for white-light-emitting diodes," Optical Materials Express, vol. 9, pp. 223-233, 2019.

[23] A. Dwivedi, et al., "Monochromatic NIR UC emission in Tm3+/Yb3+co-doped GdVO4 phosphor: the effect of the Bi3+ ion concentration and pump power of a diode laser," Optics Letters, vol. 43, pp. 5785-5788, 2018.

[24] J. S. Li, et al., "High efficiency solid-liquid hybrid-state quantum dot light-emitting diodes," Photonics Research, vol. 6, pp. 1107-1115, 2018.

[25] A. Zhang, et al., "Tunable white light emission of a large area film-forming macromolecular complex with a high color rendering index," Optical Materials Express, vol. 8, pp. 3635-3652, 2018 